\author{
J. BONEBERG ${ }^{1, \infty}$ \\ J. KÖNIG-BIRK ${ }^{1}$ \\ H.-J. MÜNZER ${ }^{1}$ \\ P. LEIDERER $^{1}$ \\ K.L. SHUFORD ${ }^{2}$ \\ G.C. SCHATZ ${ }^{2}$
}

\section{Optical near-fields of triangular nanostructures}

\author{
${ }^{1}$ University of Konstanz, 78457 Konstanz, Germany \\ 2 Department of Chemistry, Northwestern University, Evanston, IL 60208-3113, USA
}

\begin{abstract}
We compare simulations of optical near-fields of single triangular nanostructures with experimental results from a near-field ablation technique on a periodic arrangement of triangles. We find good agreement of the lateral near-field distributions; nevertheless their dependency on the polarization of the incident light differs by $90^{\circ}$. Upon increasing the lateral distances of the nanotriangle arrangement in the experiment, the polarization dependence agrees with the simulation. We conclude that this at first sight unexpected behaviour stems from the coupling of near-fields by scattered surface waves and their interaction with the incoming beam.
\end{abstract}

PACS 78.67.Bf; 81.07.-b

\section{1}

\section{Introduction}

The ongoing miniaturisation is one of the driving forces for the field of nanotechnology. In that field it would be useful to have all kinds of optics available that are frequently used on a micrometer scale, e.g., spectroscopy but also exposition of photoresists. For that purpose light must be focussed to a tiny spot well below the diffraction limit. To achieve this goal different types of nanoantennas have been proposed and examined during the last years [1-6]. For a further improvement of the efficiency of these nanoantennas progress has to be made on both sides, the theoretical description of these nanoantennas and the imaging of the near-fields with highest resolution [6-10]. The SNOM (scanning optical near-field microscopy) is an instrument used frequently, but alternative imaging methods have been developed as well which do not involve the problem of interaction of the SNOMtip with the optical near-field [11-16]. Here, we study experimental simple realizable nanoantennas, Au-triangles produced by colloidal lithography, and compare near-field simulations with the experimental results of a near-field ablation technique [14], where the optical near-field is used to pattern a silicon surface.

\section{$2 \quad$ Simulation}

The optical properties have been investigated by using the discrete dipole approximation (DDA) method for

Fax: +49-7531-883127,

E-mail: johannes.boneberg@uni-konstanz.de solving for the scattering of light from nanostructures. Details of this method have been described previously $[17,18]$. In the present application, we focus on the field intensity distribution, as defined in terms of the the square of the local electric field, $|E|^{2}$, in studies of a gold triangle on a silicon substrate. Dielectric constants for gold and for silicon are taken from Johnson and Christy [19] and Palik [20], respectively. In this calculation, the particle is discretized using a grid spacing of $3 \mathrm{~nm}$ for $240 \mathrm{~nm}$ edge length and $3.75 \mathrm{~nm}$ for $380 \mathrm{~nm}$ edge size, and the silicon substrate is treated using an effective medium approximation that uses a weighted average dielectric constant, where the weight factor is determined by the area of the particle that is exposed to the silicon. Past studies [21] have suggested that this level of discretization is capable of determining the qualitative shape of the intensity distribution in the near-field region, although absolute intensity values might be off by factors of three or more. This is adequate for the present application where absolute intensities are not important.

The simulation is performed for Au-triangles $30 \mathrm{~nm}$ in height and with side lengths of $480 \mathrm{~nm}$ and $240 \mathrm{~nm}$ on a silicon wafer. The wavelength used for all calculations is chosen to match that of the experiments, $800 \mathrm{~nm}$. This wavelength is actually well to the blue of the fundamental dipolar plasmon resonances associated with the Au-triangles. The intensity distribution is shown in the plane of the silicon surface. The simulation shows different results (Fig. 1) depending on the relative polarization of the beam with respect to the orientation of the triangle. For polarization along one baseline of the triangular structure one observes two identical peaks in the two corners along the polarization. For illumination along the perpendicular bisector of the side the intensity in one corner is about six times higher than in the other two corners. As can be seen in the lower row of Fig. 1, this behaviour does not change for the two sizes studied.

\section{$3 \quad$ Experiment}

Au-triangles are produced by the colloidal lithography technique $[22,23]$ where first a monolayer of colloidal spheres is produced on a cleaned silicon wafer. Then $30 \mathrm{~nm}$ of $\mathrm{Au}$ are evaporated onto the monolayer under high vacuum conditions. As the Au atoms reach the silicon wafer through the holes formed between the spheres only, regular triangular structures are formed on the silicon wafer. After removal of the colloidal spheres the triangular structures are illuminated 

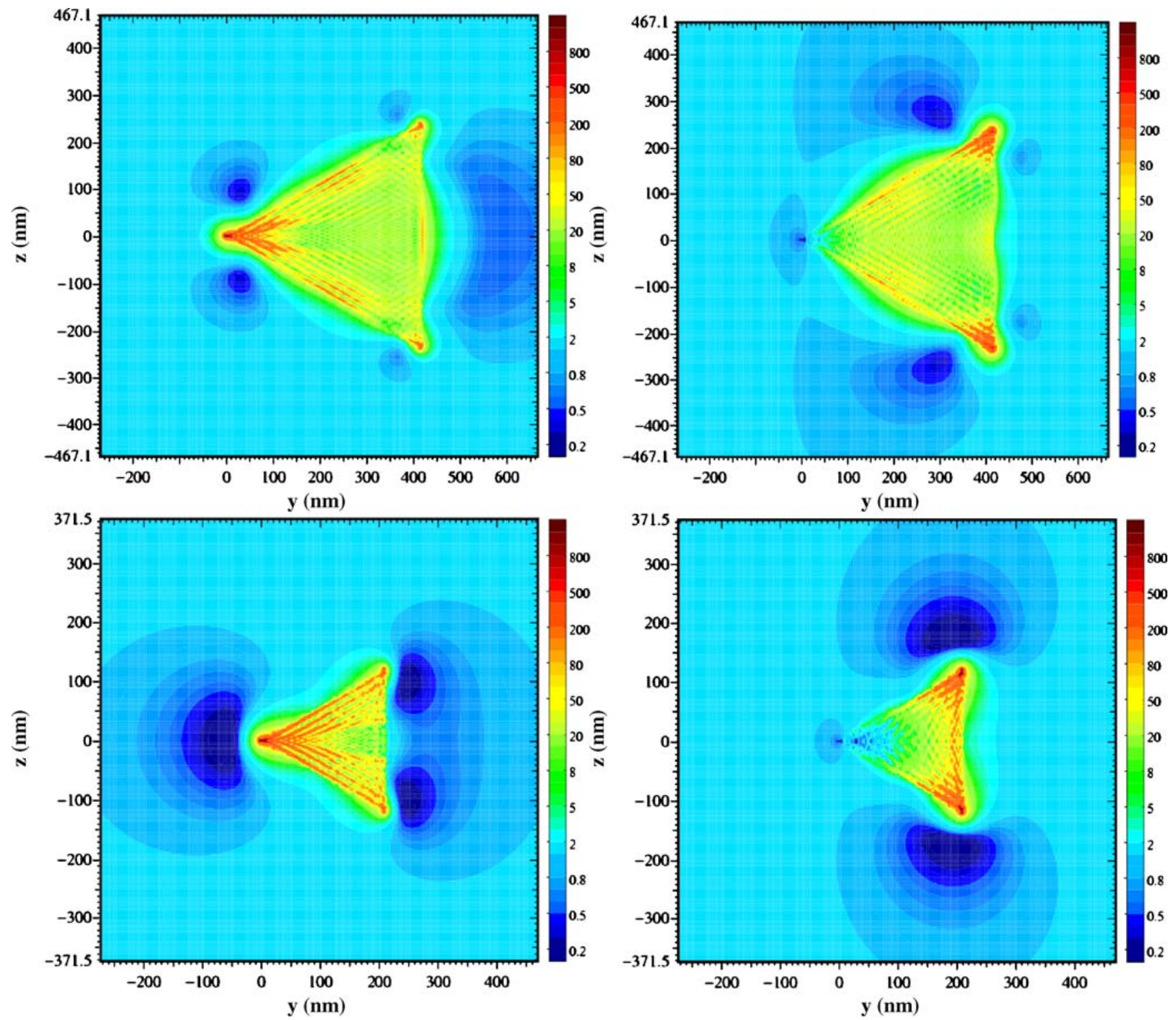

FIGURE 1 Field distribution at the interface between a Si wafer and a $480 \mathrm{~nm}$ side length triangle (upper row) and a $240 \mathrm{~nm}$ side length triangle (lower row) of $30 \mathrm{~nm}$ height $(\lambda=800 \mathrm{~nm}$, left side: polarization along $\mathrm{y}$; right side: polarization along $\mathrm{z})$

with a single intense laser pulse from a Ti-sapphire laser system $(150 \mathrm{fs}, 2 \mathrm{~mJ}, 800 \mathrm{~nm})$. The pulse is mildly focussed onto the surface in such a way that in the center of the nearly Gaussian energy distribution a small area of $\mathrm{Si}$ is ablated. Outside of the central region the energy density of the laser pulse is so low that the plain Si stays undamaged. Nevertheless, due to the optical field enhancement around the structures to be studied, local ablation takes place which reflects the optical near-field distribution. Thus it is possible to burn the near-field as an ablation pattern into the $\mathrm{Si}$ wafer. These ablation patterns can then be studied with an atomic force microscope (AFM) in detail.

Before going into the study of the near-fields of Au triangles, we would like to discuss some general features of the appearance of the near-field patterns of nanostructures. For that purpose we consider first the near-field of polysterene spheres. Figure 2 shows a calculated near-field distribution from a free space Mie-scattering calculation for a particle diameter of $800 \mathrm{~nm}(n=1.5, \lambda=800 \mathrm{~nm})$. The optical field behind the particle is enhanced by a factor of roughly ten and focussed to a diameter of half the diameter of the particle. Figure 3 shows the experimental result for a monolayer of spheres, where the energy density of the laser beam increases towards the upper left corner of the picture. Due to the pulse the particles were removed and under each original particle location a hole can be found. The diameter stays almost constant, only towards smaller energy densities of the laser pulse where the particles could not be removed the diameter gets smaller. This shows that the appearance of the nearfields in this ablation technique is quite stable. Nevertheless the holes which one would have expected are surrounded by a rim. The origin of this rim gets clearer if we consider a more complex near-field again for a spherical particle. In Fig. 4a Mie-calculations for particles of $320 \mathrm{~nm}$ diameter are shown. There the optical near-field exhibits two areas of field enhancement separated by roughly $200 \mathrm{~nm}$. The ablation pat- 

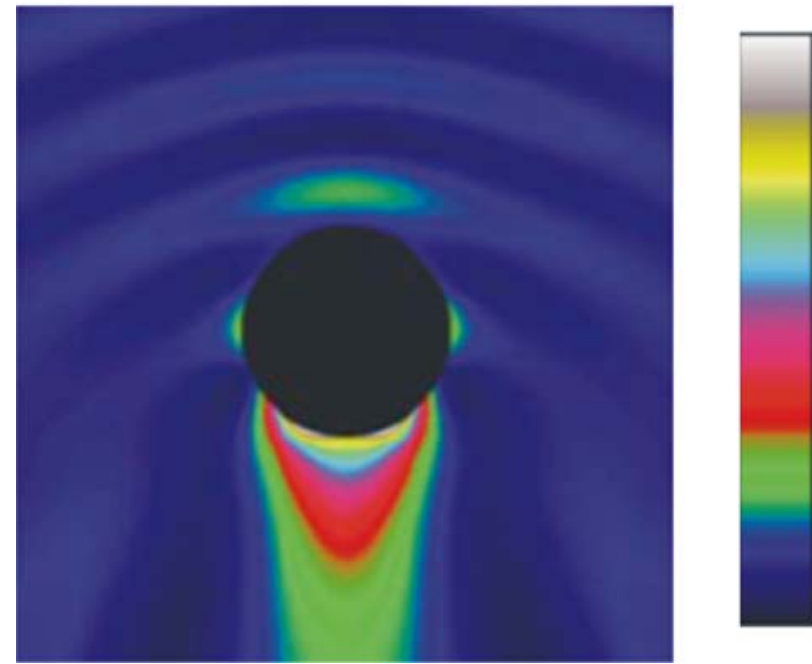

FIGURE 2 Free space Mie-calculation of the optical field distribution for a spherical polysterene particle of $800 \mathrm{~nm}$ diameter, illumination wavelength $800 \mathrm{~nm}$

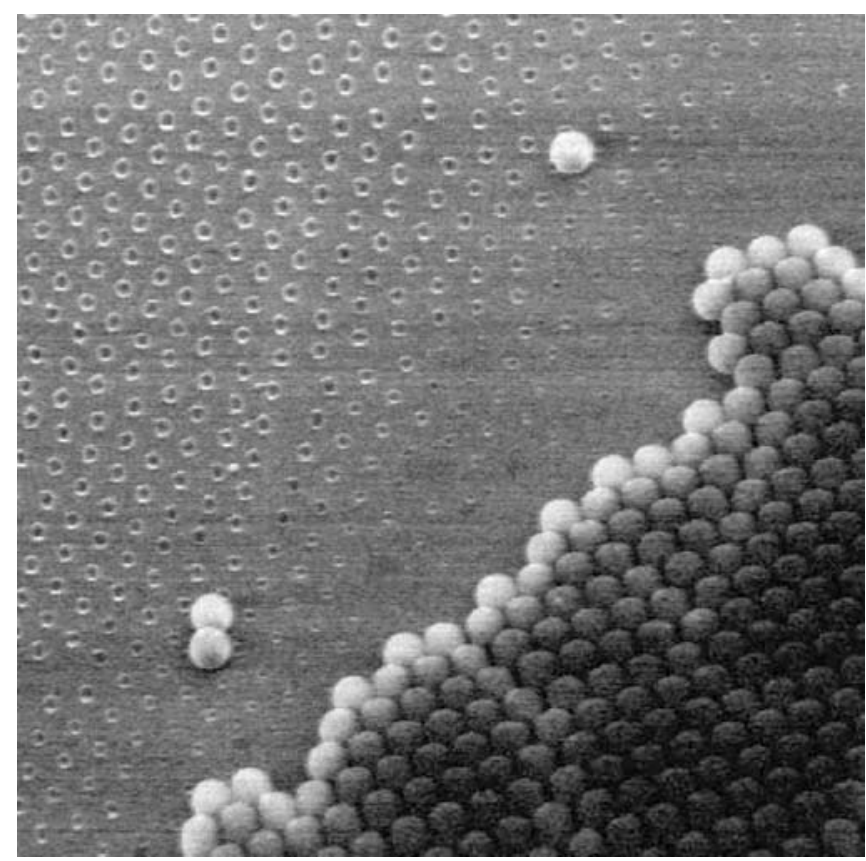

FIGURE 3 Scanning electron microscopy of a Si surface covered with $800 \mathrm{~nm}$ polysterene particles after illumination with a fs-laser pulse. The intensity of the laser pulse increases towards the left upper corner
0

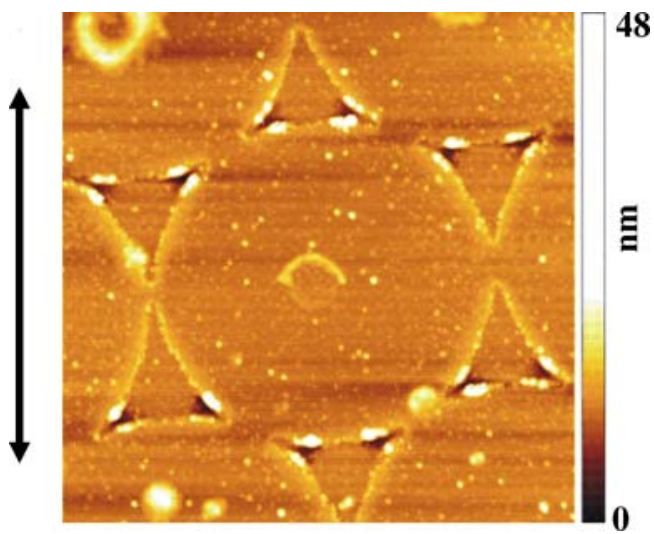

FIGURE 5 AFM-picture of the ablation pattern of an array of Au-triangles. The polarization vector direction in this case is along the ordinate axis

tern (Fig. 4b) reflects that near-field distribution, two separated areas of enhanced ablation are clearly visible, while the rim appears round. Thus the rim reflects the geometry of the particle sitting on the surface. We conclude, therefore, that the rim consists of material which is ablated and backscattered from the particle during the detachment process of the particle.

Let us now consider the near-field pattern of an arrangement of Au-triangles. Figure 5 shows an AFM-picture of a surface after the illumination with the fs-pulse. Obviously the Au-triangles (side length $480 \mathrm{~nm}$, height $30 \mathrm{~nm}$ ) disappeared; nevertheless their original position can still be identified by a narrow stripe of droplets (this stripe as well as most of the other small particles can be removed by cleaning the surface in an Au etching solution). In two of the three corners of the triangles holes are visible, which once again are accompanied by rims. The next figure (Fig. 6) shows the appearance of these triangles in an area where their orientation changed due to a grain boundary in the colloidal mask. In the smaller magnification used here, it is somewhat hard to recognize the holes in the corners, while the rims can easily be identified as a signature of the local ablation. On the right-hand side of the picture the orientation of the triangles is tilted by $90^{\circ}$. The near-field pattern of those triangles appears to be different, as the rim can be found in one of the corners only. Comparing these results with the results of the simulation (Fig. 1) we find good agreement in the pattern, but a disagreement in the dependence on polarisation: Even though the patterns look similar the dependence on polarisation differs by $90^{\circ}$.
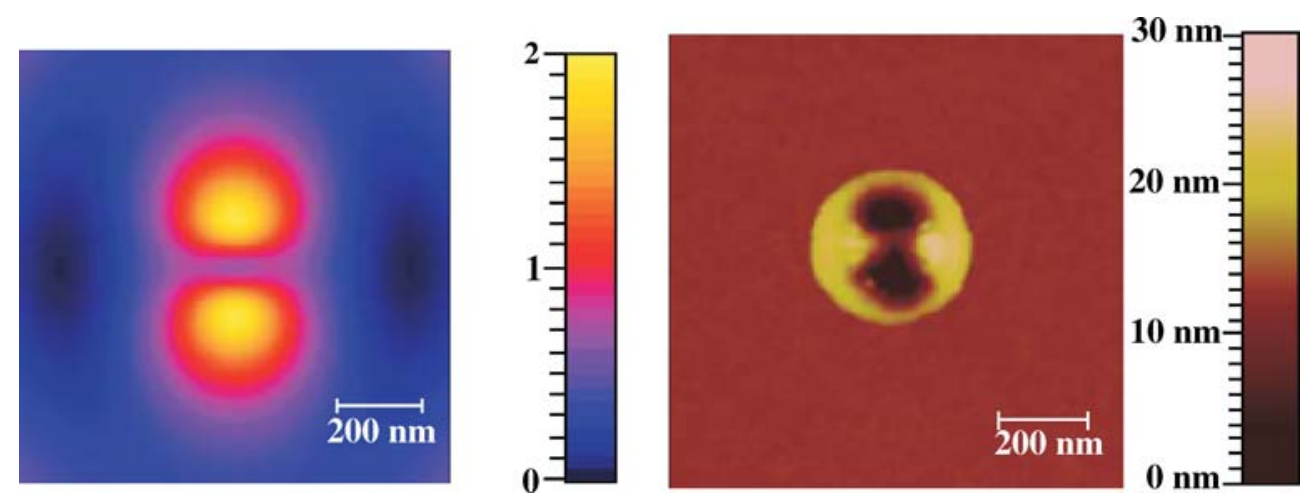

FIGURE 4 (a) Free space Miecalculation of the optical field distribution for a spherical polysterene particle of $320 \mathrm{~nm}$ diameter, illumination wavelength $800 \mathrm{~nm}$; (b) AFM-picture of the ablation pattern of a $320 \mathrm{~nm}$ polysterene particle 


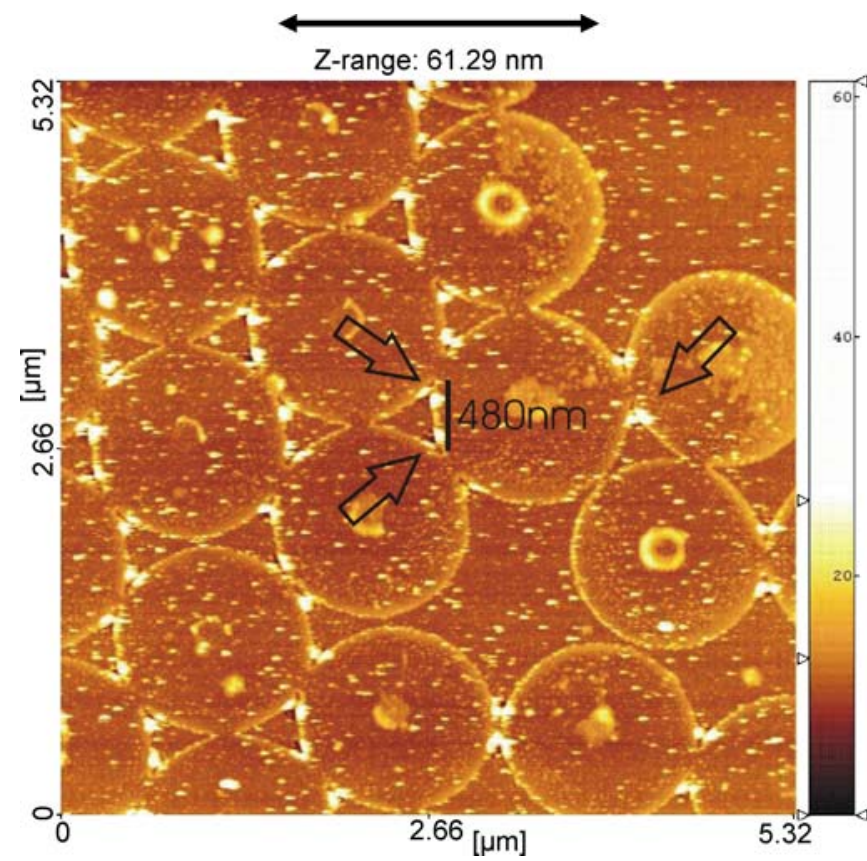

FIGURE 6 AFM-picture of the ablation pattern of an array of Au-triangles in an area with a grain boundary. The arrows in the picture indicate the different appearance of the ablation patterns. The arrow above the picture denotes the polarization of the laser pulse

At a first glance this is an astonishing difference, but both experiment and simulation were carefully reexamined and confirmed. Therefore another reason must explain this behaviour and indeed there exists a major difference between the experiment and the simulation. The simulations are performed on isolated triangles, while the experiments are performed on regular arrays of triangles. This regular arrangement might lead to a modification of the optical fields, which could be induced on one hand by coupling the near-fields directly, or on the other hand by changing the exciting field.

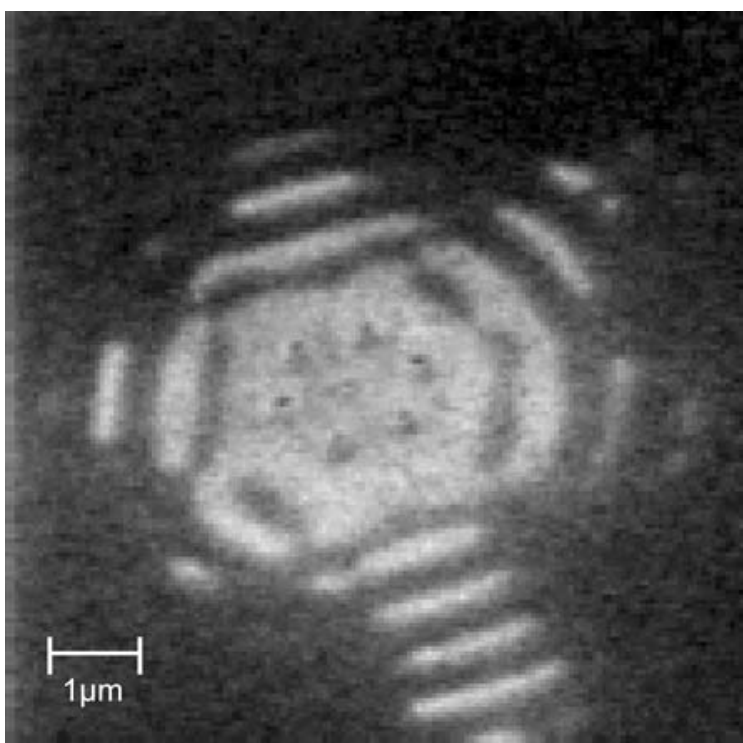

FIGURE 7 Electron microscopy of the ablation pattern induced by seven $800 \mathrm{~nm}$ polysterene particles 131006_4.001.dth

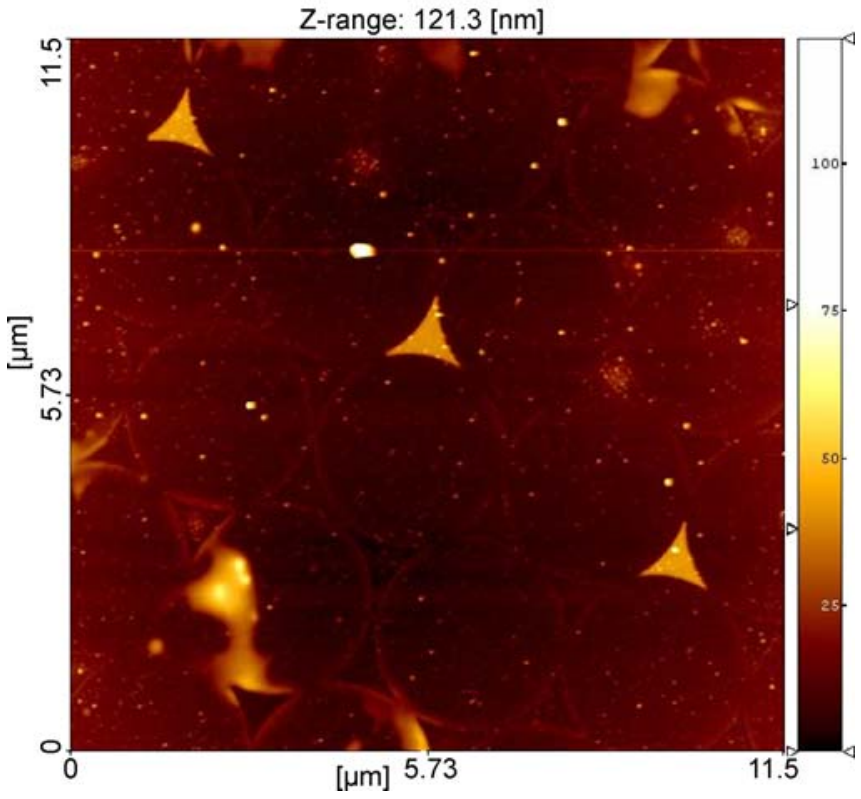

FIGURE 8 AFM-picture of isolated triangles as prepared by interfering ns-pulses

Past studies of coupling between nanoparticles have found significant effects for large particles, even when the interparticle separation is more than $10 \mathrm{~nm}$ [21]: Therefore we searched in the experiments for hints that coupling may happen over larger distances. Figure 7 shows an experiment where an arrangement of seven colloidal particles $(\mathrm{d}=800 \mathrm{~nm})$ was illuminated by the fs laser pulse. Under the original position of the particles holes can be identified, but in addition it can be seen that the surface is modified several microns outside of the particle arrangement. This result can be simulated by the in-

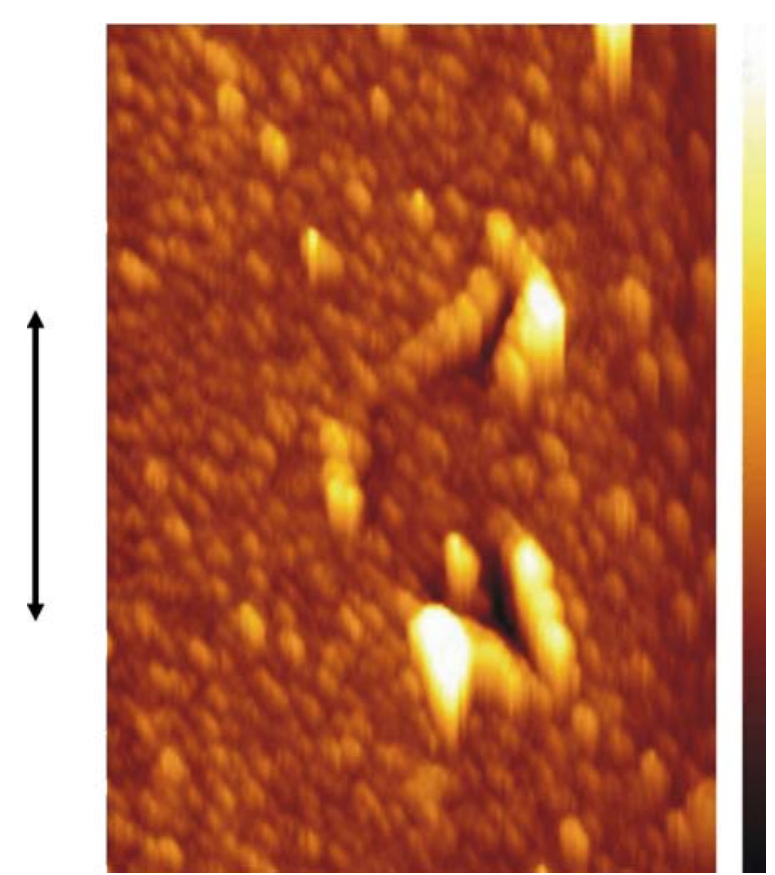

FIGURE 9 AFM-picture of the optical nearfield pattern of an isolated triangle. The polarization is indicated by the arrow 
teraction of the incoming wave with a scattered wave which propagates along the surface. Clearly the same interference effects might exist for the triangular structures and therefore the exciting field can be drastically modified. It can be expected that intensity of the scattered field depends on the size of the scatterer, a big triangle should scatter more than smaller ones.

To verify this explanation in the experiment the distance between the particles must be increased, while the shape and lateral dimension of the triangles are maintained. We prepare such samples by illumination of the original triangular arrangement with a two-beam interference pattern of a nslaser. Reaching the melting temperature, the particles undergo a dewetting process which leads finally to the ejection of the particles from the surface [24]. Figure 8 depicts the result of such a preparation process after three annealing steps under different angles. The particles are now isolated from each other. Looking at the results of the near-field patterns after a subsequent fs pulse treatment, we find in agreement with the assumption that for triangles of $240 \mathrm{~nm}$ side length the polarisation dependence agrees with the results of the simulation upon separating them by $1 \mu \mathrm{m}$ (Fig. 9) while for triangle side length $480 \mathrm{~nm}$ a separation of $950 \mathrm{~nm}$ still disagrees with simulation.

In summary we have shown that the optical behaviour of nanoantennas is strongly influenced by their surroundings. This is not only important for local near-field interaction, but interaction might also arise from an interaction of the incoming wave with a scattered wave. As a consequence the optical near-field might be modified and differ drastically from the one expected by simulations of isolated antennas.

ACKNOWLEDGEMENTS GCS and KLS were supported by a grant from the Department of Energy (DEFG02-02-ER15487). JKB and HJM were supported by the DFG via the SFB 513 and the Center of Modern Optics at the university of Konstanz.

\section{REFERENCES}

1 E.J. Sanchez, L. Novotny, X.S. Xie, Phys. Rev. Lett. 82, 4014 (1999)

2 J.P. Kottmann, O.J.F. Martin, D.R. Smith, S. Schultz, J. Microsc. 202, 60 (2001)

3 D.A. Genov, A.K. Sarychev, V.M. Shalaev, A. Wei, Nano Lett. 4, 152 (2003)

4 E. Hao, G.C. Schatz, J. Chem. Phys. 120, 357 (2004)

5 D.P. Fromm, A. Sundaramurthy, P.J. Schuck, G.S. Kino, W.E. Moerner, Nano Lett. 4, 957 (2004)

6 P. Muhlschlegel, H.-J. Eisler, O.J.F. Martin, B. Hecht, D.W. Pohl, Science 308, 1607 (2005)

7 J.R. Krenn, R. Wolf, A. Leitner, F.R. Aussenegg, Opt. Commun. 137, 46 (1997)

8 A. Bouhelier, F. Huser, H. Hamaru, H.J. Güntherodt, D.W. Pohl, F.I. Baida, D. Van Labeke, Phys. Rev. B 63, 155404 (2001)

9 M. Salerno, N. Félidj, J.R. Krenn, A. Leitner, F.R. Aussenegg, Phys. Rev. B 63, 165422 (2001)

10 R. Hillenbrand, F. Keilmann, P. Harnap, D. S Sutherland, J. Aizpurua, Appl. Phys. Lett. 83, 368 (2003)

11 P. Andre, F. Charra, P.A. Chollet, M.P. Pileni, Adv. Mater. 14, 601 (2002)

12 T. Ikawa, M. Hasegawa, M. Tsuchimori, O. Watanabe, Y. Kawata, C. Egami, O. Sugihara, N. Okamoto, Synth. Met. 124, 159 (2001)

13 P.G. Kik, S.A. Maier, H.A. Atwater, Mater. Res. Soc. Symp. Proc. 705, 101 (2002)

14 P. Leiderer, C. Bartels, J. König-Birk, M. Mosbacher, J. Boneberg, Appl. Phys. Lett. 85, 5370 (2004)

15 C. Huber, A. Rumyantseva, G. Lerondel, J. Grand, S. Kostcheev, L. Billot, A. Vial, R. Bachelot, P. Royer, A. Chang. S.K. Gray, G.P. Wiederrecht, G.C. Schatz, Nano Lett. 5, 615 (2005)

16 A. Sundaramurthy, P.J. Schuck, N.R. Conley, D.P. Fromm, G.S. Kino. W.E. Moerner, Nano Lett. 6, 355 (2006)

17 B.T. Draine, P.J. Flatau, J. Opt. Soc. Am. A 11, 1491 (1994)

18 K.L. Shuford, M.A. Ratner, G.C. Schatz, J. Chem. Phys. 123, 114713 (2005)

19 P.B. Johnson, R.W. Christy, Phys. Rev. B 6, 4370 (1972)

20 D.W. Lynch, W.R. Hunter, In: Handbook of Optical Constants of Solids, ed. by E.D. Palik (Academic Press, New York, 1985)

21 C.L. Haynes, A.D. McFarland, L. Zhao, G.C. Schatz, R.P. Van Duyne, L. Gunnarsson, J. Prikulis, B. Kasemo, M. Käll, J. Phys. Chem. B 107, 7337 (2003)

22 U.C. Fischer, H.P. Zingsheim, J. Vac. Sci. Technol. 19, 881 (1981)

23 F. Burmeister, W. Badowsky, T. Braun, S. Wieprich, J. Boneberg, P. Leiderer, Appl. Surf. Sci. 145, 461 (1999)

24 A. Habenicht, M. Olapinski, F. Burmeister, P. Leiderer, J. Boneberg, Science 309, 2043 (2005) 